\title{
Políticas nacionais e o campo da Alimentação e Nutrição em Saúde Coletiva: cenário atual
}

\author{
National policies and the field of Food and Nutrition \\ in Collective $\mathrm{H}$ ealth: the current scenario
}

Elisabetta Recine ${ }^{1}$

Ana Beatriz Vasconcellos ${ }^{2}$

\footnotetext{
${ }^{1}$ Observatório de Políticas deSegurança Alimentar e Nutrição, Departamento de Nutrição, Faculdadede Ciências da Saúde, Universidade de Brasília. Campus Universitário Darcy Ribeiro, Asa N orte. 70910-900 Braślia DF. recine@unb.br

${ }^{2}$ Coordenação Geral da Política de Alimentação e Nutrição, Departamento de Atenção Básica, Secretaria deAtenção à Saúde, M inistério da Saúde.
}

Abstract I t ispresented a review of the guidelines implementation of the $\mathrm{N}$ ational Food and $\mathrm{N}$ utrition Policy (PNAN) contextualizing the actions in the Brazilian Unified Health System (SUS) scenario. At ten years of its publication, PNAN faces challenges both to expand and qualify the shares of food and nutrition on health. It is challenging to stand as interlocutor and legitimate representative of the area of health, political and institutional context of food security and nutrition. Issues related to the articulation of PNAN and future National Policy on Food and N utrition Security will be analyzed to demonstrate the convergence of agendas among the priorities for the guarantee of the SAN. The authors identify the potential of this field of action, from the current institutional setting, and the need for comprehensive solutions that address the complexity of food and nutrition in health.

Key words Food and Nutrition Policy, Health System, Food and nutrition security, Collective Health
Resumo É apresentado um balanço da implementação das diretrizes da Política Nacional de Alimentação e N utrição (PNAN) contextualizando as ações no cenário do Sistema Ú nico deSaúde (SUS). A os dez anos desua publicação, a PNAN se defronta com desafios tanto para expandir como para qualificar as ações de alimentação e nutrição na saúde, e também para se apresentar como interlocutora e representante legítima da área da saúde, no contexto político e institucional da segurança alimentar e nutricional. Q uestões relacionadas à articulação da PN AN e da futura Política N acional de Segurança Alimentar e N utricional são anali sadas para demonstrar a confluência de agendas entre as prioridades para a garantia da SAN . São apontadas aspotencialidades desse campo de ação, a partir do atual cenário institucional, e a necessi dade de soluções abrangentes que atendam à complexidade da alimentação e nutrição em Saúde Coletiva.

Palavras-chave Política Nacional de Alimentação e Nutrição, Sistema Único de Saúde, Segurança alimentar e nutricional, Saúde Coletiva 


\section{A Política Nacional deAlimentação e Nutrição hoje}

As políticas desenvolvidas pelo Estado brasileiro, cujo objeto envolve as dimensões múltiplas da alimentação e nutrição, ganharam novo dire cionamento a partir da Lei Orgânica de SegurançaAlimentar N utricional (Losan) - Lei no 11.346, de 15 de setembro de 2006, que criou o Sistema N acional de Segurança Alimentar e Nutricional $(\text { Sisan })^{1}$. De acordo com o conceito adotado na legislação brasileira, A segurança alimentar e nutricional - SAN - consiste na realização do direito de todos ao acesso regular e permanente a alimentos de qualidade, em quantidade suficiente, sem comprometer 0 acesso a outras necessidades essenciais, tendo como base práticas alimentares promotoras de saúde que respeitem a diversi dade cultural e sejam ambiental, cultural, econômica e socialmentesustentáveis (art. 3o da Lei no 11.346 , de 15 de setembro de 2006) ${ }^{1}$.

A dimensão nutricional do conceito incorpora questões relativas à composição, à qualidade, à utilização biológica e à promoção da saúde, apontando a abrangência das políticas que convergem para 0 alcance da segurança alimentar e nutricional. Entreelas, está a Política Nacional de Alimentação e Nutrição (PNAN) ${ }^{2}$ como elo potencial entreo Sistema Ú nico de Saúde $(S U S)^{3}$ eo Sistema Nacional de Segurança Alimentar e Nutricional (Sisan) ${ }^{1}$.

A Política Nacional de Alimentação e Nutrição, publicada no final dos anos 90 , marca uma etapa importante para a configuração da área no campo da saúde, evidenciando as transições epidemiológica, nutricional edemográfica com a convivência no país de situações extremas de desnutrição e deficiências nutricionais ao lado de altas prevalências de obesidade e doenças associadas à alimentação. A PNAN ${ }^{2}$ projeta um modelo de segurança alimentar enutricional fundamentado no direito humano à alimentação, destacando a alimentação e a nutrição como requisitos de promoção e proteção da saúde 2 .

Reposicionando, naépoca, a questão alimentar enutricional na agenda das políticas públicas do setor saúde, a PNAN ${ }^{2}$ assume o propósito, com o estímulo a ações intersetoriais, de garantir a qualidade dos alimentos consumidos no país, promover práticas alimentares saudáveis, prevenir e controlar distúrbios nutricionais. A Política articula sete diretrizes, que orientam a elaboração e implantação dos programas e projetos em alimentação enutrição: estímulo às ações intersetoriais com vistas ao acesso universal aos alimentos; garantia da segurança e da qualidade dos alimentos e da prestação de serviços; monitoramento da situação nutricional e alimentar; promoção de práticas alimentares eestilos devida saudáveis; prevenção e controle dos distúrbios nutricionais e de doenças associadas à alimentação e nutrição; promoção do desenvolvimento de linhas de investigação; e desenvolvimento e capacitação de recursos humanos².

As características da implementação de uma política revelam seu verdadeiro significado, eo processo percorrido pela PNAN ${ }^{2}$ não édiferente, ensejando desafios para os profissionais, pesquisadores e gestores. Estudo realizado pelo M inistério da Saúde acerca do desempenho da PNAN ${ }^{2}$ demonstrou seu papel de referência regulatória, política, técnica e ética para os profissionais de nutrição quetrabal ham naárea da SaúdeColetiva. São considerados méritos: a configuração e o desenvolvimento da vigilância alimentar enutricional, a produção regular de informações sobre estado nutricional, por meio de pesquisas de base populacional, a construção da agenda de promoção da alimentação saudável e a capacitação de recursos humanos. Permanecem desafios importantesa institucionalidade da área, a organização do processo de trabalho, o financiamento e o controle social ${ }^{4}$.

No âmbito da vigilância alimentar enutricional, o aperfeiçoamento e a expansão do Sistema de Vigilância Alimentar e Nutricional (Sisvan) ${ }^{5}$ respondem aos esforços de programar um sistema operativo na rotina das unidades de saúde que possa favorecer o planejamento racional de ações do nível local, contribuindo para efetividade da PNAN ${ }^{2}$. As medidas sensíveis da fome e seus determinantes, da desnutrição, da deficiência nutricional resultante de diversas causas, da mudança do perfil de consumo de alimentos e consequências como excesso de peso e obesidade são indicadores de como a sociedade atravessa as etapas de desenvolvimento social eeconômico e incorpora ou abandona padrões de consumo de alimentos e práticas de saúde. M onitorar a situação de saúde, alimentar e nutricional de maneira sistemática revela desigualdades entre regiões geográficas, grupos populacionais e populações vulneráveis e permite subsidiar intervenções voltadas para a prevenção e o controle de distúrbios nutricionais e doenças associadas à alimentação e nutrição e para a promoção de práticas alimentares e estilos de vida saudáveis. Para tal, permanece como desafio a construção de indicadores cujo conteúdo informativo associado seja capaz de incorporar as dimensões das iniquidades em saúde 6 . 
O Sisvan tem sua base de informações oriunda da rotina da atenção básica de saúde, em especial das equipes de saúde da família, eé atualmente utilizado para medir os resultados alcançados pelos gestores de saúde das esferas estadual emunicipal no cumprimento das metas dealimentação e nutrição assumidas no Pacto N acional de Saúde. Os dados gerados pelo Sisvan informam o perfil nutricional de uma dada população vinculada geograficamentea uma determinada unidade de saúde, consolidando sua vocação como sistema de informação de base local para a segurança alimentar e nutricional ${ }^{5,7}$.

A realização de pesquisas nacionais periódicas compõeo sistema demonitoramento alimentar enutricional. Nosúltimos cinco anos, foram realizadas pesquisas importantes para o campo da alimentação e nutrição em Saúde Coletiva. A Pesquisa Nacional de Demografia eSaúdeincluiu o primeiro estudo nacional sobre a prevalência de anemia e hipovitaminose A; as Pesquisas de Orçamento Familiar dos anos de 2008 e2009 trouxeram como inovação a incorporação de um módulo sobre o consumo direto de alimentos, 0 que irá atualizar, após mais de três décadas, em âmbito nacional, dados de consumo. A Pesquisa Nacional de Avaliação do Impacto da Iodação do Sal, elaborada nos moldes propostos pela Organização M undial da Saúde, poderá incluir o Brasil na relação de países que eliminaram as doenças por carência de iodo.

A agenda de promoção da al imentação saudável foi qualificada com a edição do Guia alimentar para a população brasileira ${ }^{8}$, que assumeo conceito de alimentação saudável como resultado da interação entre o biológico e o sociocultural. A abordagem multifocal dirigida às famílias, aos profissionais de saúde, aos gestores e formuladores de políticas públicas e setor produtivo de alimentos ilustra como atuar nos fatores determinantes da alimentação, explicitando as responsabilidades dos diferentes setores sociais, indicando que apenas ações de educação em saúde não são suficientes para o alcance do impacto necessário na promoção da alimentação saudável. São definidas medidas regulatórias referentes à rotulagem de alimentos eà publicidade dealimentos com altos conteúdos de açúcar, gordura e sal; que orientam o eixo de alimentação saudável proposto na Política Nacional de Promoção da Saúde e eas ações de avaliação nutricional e promoção da saúde do Programa Saúde na Escola ${ }^{10}$, programa conjunto do M inistério da Saúde e do M inistério da Educação.

Estudo realizado ${ }^{11}$ mostra 0 quanto 0 setor saúde pode, com base no processo de troca di- nâmica de informações sobre alimentação e nutrição, contribuir para a formação da opinião confiável sobre os princípios e recomendações da alimentação sau dável. E neste contexto, a atenção básica se constitui em um locus privilegiado para se avançar na promoção da alimentação saudável ${ }^{12}$.

Os conceitos e recomendações do Guia ${ }^{8}$ foram ainda referência para a proposta de Programa Intersetorial de Promoção da Alimentação Adequada e Saudável apresentada pelo ConseIho Nacional deSegurançaAlimentar eN utricional ${ }^{13}$. Esta convergência em torno de uma agenda de promoção da alimentação adequada e saudável configura-se como elemento de diálogo propositivo entre movimentos da sociedadecivil de SAN e de Saúde Coletiva.

A criação dos Núcleos de Apoio à Saúde da Família (NASF) ${ }^{14}$, com o objetivo de ampliar a abrangência e o escopo das ações da atenção básica, bem como sua resolutividade, é passo importante para impulsionar a organização das ações de nutrição nos serviços de saúde. Entreas atribuições do nutricionista no N ASF encontramse: o cuidado nutricional no curso da vida, as respostas às principais demandas assistenciais (distúrbiosalimentares, deficiências nutricionais e desnutrição), os planos terapêuticos nas doenças crônicas; conhecimento e estímulo à produção e ao consumo dos alimentos saudáveis; a articulação intersetorial para viabilizar hortas e pomares comunitários; a organização da referência e contrarreferência do atendimento. A incorporação do nutricionista para prestar apoio matricial às equipes de saúde da família no desenvolvimento de atribuições amplas, desde 0 diagnóstico coletivo ao cuidado individual, deve aumentar a demanda por qualificação profissional em Saúde Coletiva, implicando adequação futura dos cursos de graduação em nutrição.

No processo de capacitação de recursos humanos, muito se deve aos Centros Colaboradores de Alimentação e Nutrição do M inistério da Saúde. Compostos por dez universidades federais (Universidade Federal do Pará, Universidade Federal de Pernambuco, Universidade Federal da Bahia, Universidade Federal de M inas Gerais, Escola Nacional deSaúdePública Sergio Arouca, Fundação O swaldo Cruz/Ministério da Saúde, Universidade de São Paulo, Universidade Federal do Paraná, Universidade Federal de Pelotas, Universidade de Brasília, Universidade Federal de Goiás), esses centros configuram uma rede de apoio às ações de alimentação em nutrição nas cinco regiões brasileiras buscando integrar ensino, pes- 
quisa e serviço. Com esse marco de atuação, os centros, de forma autônoma, contribuem para disseminação dos princípios do SU Se daPNAN e da atenção primária em saúde; participam em projetos de pesquisas multicêntricas em saúde e nutrição, contribuem na análise dos bancos de dados de pesquisas nacionais, aprofundando a análise regional, sub-regional ou local, e promovem o conhecimento e a avaliação de programas e políticas de segurança alimentar e nutricional. Ressalta-se a liderança em projetos de pesquisas nacionais e a vocação para renovação de quadros para a área de nutrição e Saúde Coletiva.

0 apoio a linhas de pesquisa e estudos em áreas estratégicas do conhecimento em nutrição foi viabilizado por meio da publicação de dois editais nacionais. 0 Edital sobre Alimentação e Nutrição foi lançado no ano de 2004 em conjunto pelo M inistério da Saúde e o Conselho Nacional de Desenvolvimento Científico e Tecnológico (CN Pq). Este edital, cujas linhas temáticas estão voltadas às diretrizes da PNAN, gerou o financiamento de 85 projetos, com um investimento que ultrapassou os $\mathrm{R} \$ 4$ milhões, fato inédito no campo da al imentação enutrição no Brasi| ${ }^{15}$. Em 2005, - Edital Alimentação, Nutrição e Promoção da Alimentação eM odos de Vida Saudáveis, lançado de forma similar ao anterior, pelo Ministério da Saúdeeo CN Pq, apoiou 97 projetosem diferentes áreas temáticas, incluindo as intervenções em nutrição e Saúde Coletiva, buscando consolidar um modelo de fomento à pesquisa. A avaliação deste processo apontará em que medida os institutos de pesquisa e universidades brasileiras irão trazer inovações e consolidá-las para um maior diálogo entrea academia eas políticas públicas dealimentação e nutrição em saúde e oportunizar, tanto no âmbito acadêmico quanto no sistema de saúde, investigações e pesquisas que aprimorem o caminho da nutrição nas políticas públicas brasileiras em seu sentido abrangente eintersetorial.

A futura Política Nacional de Segurança Alimentar e Nutricional

Com a sanção da Losan, em 2006, fica instituído - Sistema Nacional de SAN (Sisan) ${ }^{1}$, que tem como objetivos: (1) a formulação da Política Nacional de Segurança Alimentar e Nutricional (PNSAN) nas diferentes esferas degoverno; e(2) a inclusão de princípios e diretrizes de segurança alimentar enutricional nas políticas referentes às áreas abrangidas pela definição de SAN adotada no Brasil. Particularmente em relação à área da saúde, a Losan explicita, em seu artigo 6우 que a SAN abrange a promoção da saúde, da nutrição e da alimentação da população, incluindo-se grupos populacionais espeć́ficos e populações em situações de vulnerabilidade social" ${ }^{\prime}$.

A partir da III Conferência Nacional deSAN, realizada em 2007, o Consel ho Nacional de SAN (Consea) instituiu um processo de elaboração, com base nas resoluções finais do encontro, de um documento com os princípios ediretrizes que deverão compor a Política Nacional de Segurança Alimentar e Nutricional. As resoluções finais da CNSAN ${ }^{16}$ foram organizadas em três eixostemáticos: SAN nas estratégias nacionais de desenvolvimento; Política Nacional deSAN; eSistema Nacional de SAN. No segundo eixo foram propostas seis diretrizes: (1) promover o acesso universal à alimentação adequada; (2) estruturar sistemas justos, de base agroecológica esustentável deprodução, extração, processamento e distribuição de alimentos; (3) instituir processos permanentes de educação e capacitação em segurança al imentar e direito humano à alimentação adequada; (4) ampliar e coordenar as ações de segurança alimentar e nutricional voltadas para povos indígenas e comunidades tradicionais; (5) fortalecer as ações de alimentação e nutrição em todos os níveis da atenção à saúde, de modo articulado às demais políticas de segurança alimentar e nutricional; (6) promover a soberania e a segurança alimentar enutricional em âmbito internacional.

0 documento foi enviado à Câmara Interministerial de SAN (CAISAN) ${ }^{17,18}$ em outubro de 2009. A CAISAN, composta pelos ministros ou seus representantes, atualmente, de 19 áreas de governo, é responsável por propor e articular a implementação da PNSAN.

Neste documento do Consea, "Subsídios para a construção da Política Nacional de Segurança Alimentar e Nutricional"17, está proposto que 0 objetivo geral da PNSAN é assegurar o direito humano à alimentação adequada a todas e todos os habitantes do território brasileiro, promovendo a soberania e a segurança alimentar e nutricional de modo que tenham acesso regular e permanente a alimentos dequalidade, em quantidade suficiente, sem comprometer 0 acesso a outras necessidades essenciais, tendo como base práticas alimentares promotoras de saúde, que respeitem a diversidade cultural e que sejam ambiental, cultural, econômica e socialmente sustentáveis.

A quinta diretriz referente ao campo da alimentação e nutrição no âmbito da saúde, "Fortalecer as ações de alimentação e nutrição em todos os níveis de atenção à saúde, de modo arti- 
culado às demais políticas de SAN", resgata a "Promoção da Alimentação Saudável" como eixo estruturante de todas as ações a serem efetivadas pela PNSAN ${ }^{17}$, entendendo que a promoção da alimentação saudável compreende todas as etapas do ciclo do alimento (da produção ao consumo) e deve ser implementada em todas as fases do curso da vida eníveis de atenção em saúde edemaneira articulada com diferentes setores de governo e da sociedade.

As ações derivadas dessa diretriz deverão impactar: (1) na promoção do acesso a uma alimentação adequada e saudável; (2) na qualidade sanitária enutricional dos alimentos e da alimentação; (3) na integração de programas que contribuem na melhoria do acesso à alimentação; (4) no acompanhamento da situação alimentar enutricional da população brasileira; (5) na produção do conhecimento sobre alimentação enutrição; (6) na visibilidade, valorização efortalecimento da área de nutrição no SUS; (7) na elaboração de políticas de alimentação enutrição para povos e comunidades tradicionais; (8) na concepção de programas para pessoas com anemia falciforme e outras necessidades al imentares especiais; e (9) na implementação e ampliação do controlesocial.

Considerando a "Promoção da Alimentação Saudável" (PAS) como eixo estruturante da PN SAN e as repercussões que essa decisão trará para todas as áreas relacionadas com a SAN, 0 fortalecimento das ações de alimentação e nutrição no Sistema Único de Saúde (SUS) adquire uma importância estratégica. A dimensão ampliada de PAS conferida pelo conjunto de proposições da III CNSAN destacou a Política PNAN ${ }^{17}$ como elemento fundamental para a construção dessa trajetória, em razão de ela articular ações que reafirmam a segurança alimentar e nutricional e 0 direito humano à alimentação adequada como valoresindissociáveis da promoção da saúde e da cidadania. É, portanto, necessário que tais valores perpassem as ações de promoção, atenção e vigilância à saúde. A presentam-se também como estratégicas a disseminação deinformações no campo da alimentação saudável e da nutrição, a importância da formação profissional qualificada e específica para atuação nessa área e a ne cessidade de uma educação permanente dos profissionais desaúde. A vigilância da situação nutricional da população brasileira e o estabelecimento de um controle social democrático dessa política, em suas diferentes instâncias de execução, foram também considerados questões fundamentais para 0 avanço desse processo.
N este contexto, são destacados como imprescindíveis o fortalecimento ea institucionalização da área e das ações de al imentação e nutrição no interior do SUS, recuperando os eixos da PNAN ${ }^{17}$ e incentivando sua capacidade de construir diálogos intra e intersetoriais. É, ainda, explicitado que o fortalecimento necessário e concreto da PNAN devese refletir no financiamento adequado para a implementação de suas diretrizes.

Com a inserção, a regulamentação e a organização das ações de nutrição em todos os níveis de atenção à saúde, o setor saúde poderá atuar de maneira qualificada na perspectiva de uma atenção nutricional abrangente, inclusive em relação à promoção da saúde, contribuindo para a mudança de paradigma do modelo de atenção à saúde como um todo e contribuindo efetivamente para a garantia da SAN.

Como consequência desse processo, a CAISAN elaborou uma proposta de projeto de lei sobre a PNSAN ${ }^{17}$. A proposta foi discutida pela plenária do Consea em dezembro de 2009 e está em fase de aprimoramento e definição dos trâmites para envio ao Congresso $\mathrm{N}$ acional.

\section{Possibilidades de cenário futuro}

A PN AN ${ }^{17}$ completou dez anos e acumulou um conjunto importante de conquistas mas também de desafios apontados no documento de subsídio ${ }^{19}$ para os seminários estaduais de alimentação e nutrição no SUS, etapa do processo de discussão eformulação derecomen dações parauma nova edição do texto. A PNAN foi elaborada a partir de evidências epidemiológicas fortes - que nortearam a argumentação e a definição de suas diretrizes. A pesar deser formulada em uma época defortalecimento dos argumentos por um "estado mínimo", valorizou a intersetorialidade, contextualizou-secomo el emento de contribuição da SAN e adotou o Direito Humano à Alimentação Adequada como princípio. Além disso, de vários pontos de vista, a Política antecipou várias recomendações presentes posteriormente na Estratégia Global para a Atividade Física e Dieta ${ }^{20}$.

No momento em que, por um lado, deflagra-se um processo organizado de balanço de sua implementação e, por outro, destacam-se aspectos em relação ao papel da alimentação e nutrição na saúde na SAN, é imprescindível o compromisso de identificar os desafiose lacunas e as estratégias que atendam a eles. 0 detalhamento das instâncias e o modus operandi do Sisan somam aspectos concretos aos já identifica- 
dos ao longo desta década de implementação da PNAN. Hánecessidade de serem respondidas algumas questões: como e o quanto a Saúde se vê no campo da SAN, sua contribuiç̧ão, seu papel, tanto no desenvolvimento de ações como também disputando e decidindo agenda; como e o quanto aSAN vêa saúde como parceira legítima, como se articulam esses espaços políticos einstitucionais; como os dois sistemas, SU S e Sisan, se articulam em suas prioridades, agendas e processos; como a PNAN e a futura PNSAN ${ }^{17}$ se articulam e se coordenam.

No âmbito da saúde, são inadiáveis a expansão e a qualificação das ações de alimentação e nutrição no Sistema Ú nico de Saúde, para que a área da saúde ocupe e responda por suas responsabilidades no desafio de promover a SAN da população brasileira. Este esforço deve ser acompanhado por uma profunda reflexão eade quação da formação dos profissionais que atuam em nutrição em Saúde Coletiva para que estes se coloquem em uma posição de formulação eliderança.

Há claramente uma confluência de agendas entre as prioridades para a garantia da SAN e oS avanços necessários para a efetiva implementação e atualização das ações de alimentação e nutrição no SUS- representadas pela PNAN ${ }^{17}$. Cabe compartilhar estratégias para que os avanços sejam efetivos e ágeis.

Em suma, o investimento político e programático no campo da alimentação e nutrição em Saúde Coletiva configura-se como uma janela de oportunidades importante em um cenário no qual explicações da realidade e busca de soluções precisam ser abrangentes para responder às novas complexidades da nutrição e da inserção da alimentação na esfera dos direitos humanos.

\section{Colaboradores}

E Recine e $A B$ Vasconcellos foram responsáveis pela concepção eelaboração do texto. As autoras agradecem a Paula Jeane Araújo pela revisão das referências bibliográficas. 


\section{Referências}

1. Brasil. Lei Orgânica de Segurança Alimentar Nutricional (Losan). Lei no 11.346, de 15 de setembro de 2006. Cria o Sistema Nacional de Segurança Alimentar e Nutricional-SISAN com vistas em assegurar 0 direito humano à alimentação adequada e dá outras providências. Diário O ficial da U nião 2006; 18 set. [acessado 2010 jul 16]. Disponível em: https:// www.planalto.gov.br/ccivil_03/_Ato2004-2006/2006/ Lei/L11346.htm

2. Brasil. Ministério da Saúde. Portaria no 710 , de 10 de junho de 1999. Aprova a Política Nacional de Alimentação e Nutrição-PNAN e dá outras providências. Diário Oficial da U nião 1999; 11 jun.

3. Brasil. Lei no 8.080, de 19 de setembro de 1990. Lei Orgânica do SUS: Dispõe sobre as condições para a promoção, proteção e recuperação da saúde, a organização e o funcionamento dos serviços correspondentes e dá outras providências. Diário 0 ficial da União 1990; 20 set.

4. Brasil. M inistério da Saúde/Organização Pan-Americana da Saúde. PNAN 3 tempos: uma análise do processo de implementação da Política Nacional de Alimentação e Nutrição [relatório de pesquisa]. Brasília: M inistério da Saúde/O pas; 2009.

5. Brasil. M inistério da Saúde. Instituto Nacional de Alimentação e Nutrição. Portaria no 1.156, de 31 de agosto de 1990. Institui, no M inistério da Saúde, o Sistema de Vigilância Alimentar e Nutricional-SISVAN e dá outras providências. Diário Oficial da União 1990; 31 ago.

6. M agal hães R. M onitoramento das desigualdades sociais em saúde: significados e potencialidades das fontes de informação. Cien Saude Colet 2007; 2(3):667-673.

7. Engstrom EM, Castro IRR. Monitoramento em nutrição e saúde: articulação da informação com a ação. Saude Rev 2004; 6(13):45-52.

8. Brasil. M inistério da Saúde. Secretaria de Atenção à Saúde. Departamento de Atenção Básica. Guia alimentar para a população brasileira: promovendo a alimentação saudável. Brasília: M inistério da Saúde; 2008. (Série $A$, Normas e M anuais Técnicos).

9. Brasil. M inistério da Saúde. Secretaria de Vigilância em Saúde. Secretaria de Atenção à Saúde. Política Nacional de Promoção da Saúde. Brasília: Ministério da Saúde; 2006. (Série B, Textos Básicos de Saúde).

10. Brasil. Decreto Presidencial no 6.286, de 5 de dezembro de 2007. Institui o Programa Saúde na Escola (PSE), e dá outras providências. Diário Oficial da União 2007; 6 dez.

11. Silva DO, Recine EGIG, Queiroz EFO. Concepções de profissionais de saúde da atenção básica sobre a alimentação saudável no Distrito Federal, Brasil. Cad Saude Publica 2002; 18:1367-1378.

12. Vasconcelos ACCP, Pereira IDF, Cruz PJSC. Práticas educativas em nutrição na atenção básica em saúde: reflexões a partir de uma experiência de extensão popular em João Pessoa-Paraíba. Rev APS 2008; 11(3):334-340.
13. Brasil. Conselho Nacional de Segurança Alimentar e Nutricional. Grupo Técnico de Alimentação Adequada e Saudável [relatório final], 2007. [acessado 2010 jul 16]. Disponível em: https://www.planalto. gov.br/Consea/static/documentos/Tema/Alimentacao Adequa/RelatorioFinal.pdf

14. Brasil. M inistério da Saúde. Portaria no 154, de 24 de janeiro de 2008. Cria os Núcleos de Apoio à Saúde da Família-NASF, e dá outras providências. Diário Oficial da União 2008; 4 mar.

15. Kac G, Serruya S. Epidemiologia nutricional materno-infantil e a agenda de prioridades de pesquisa em saúde no Brasil. Cad Saude Publica 2008; 24(2):s206-s207.

16. Brasil. Conselho Nacional de Segurança Alimentar e Nutricional. III Conferência Nacional de Segurança Alimentar [relatório final]. Brasília: Consea; 2007. [acessado 2010 jul 16]. Disponível em: http:// www. planalto.gov.br/consea/3conferencia/static/ Documentos/RelatorioFinal.pdf

17. Brasil. Conselho Nacional de Segurança Alimentar e Nutricional. Subsídios para a Construção da Política N acional de Segurança Alimentar e Nutricional, 2009. [acessado 2010 jul 16]. Disponível em: http:// www.planalto.gov.br/consea/static/documentos/ Tema/Losan/D ocumento\%20CP3\%20PN SAN \% 20 final.pdf

18. Brasil. Câmara Interministerial de Segurança Alimentar e Nutricional. Resolução n 01 , de 23 de junho de 2009. Subsídio para Balanço das Ações Governamentais de Segurança Alimentar e Nutricional e da Implantação do Sistema Nacional. Documento elaborado para o encontro: III Conferência Nacional de Segurança Alimentar e Nutricional+2. Brasília: CAISAN; 2009. [acessado 2010 jul 16]. Disponível em: https://www.planalto.gov.br/consea/static/eventos/ III conferencia+2/D ocumentos/Balan \%E 70SAN Final.pdf

19. Brasil. Conselho Nacional de Segurança Alimentar e Nutricional. Comissão Intersetorial de Alimentação e Nutrição. Documento base de subsídio do Seminário Estadual de Alimentação e Nutrição no SUS, 2010. [acessado 2010 jul 16]. Disponível em: http://nutricao.saude.gov.br/docs/geral/documento base_semi_estadual.pdf

20. World Health Organization. Global strategy on diet, physical activity and health. Geneva: WH O; 2004.

Artigo apresentado em 10/02/2010

Aprovado em 20/05/2010

Versão final apresentada em 12/07/2010 